\title{
Statistical Modeling of Large-Scale Simulation Data
}

\author{
T. Eliassi-Rad, T. Critchlow, G. Abdulla
}

This article was submitted to

The Eighth ACM SIGKDD International Conference of Knowledge Discovery and Data Mining, Edmonton, Alberta, Canada, July 23-26, 2002

\section{February 22, 2002}

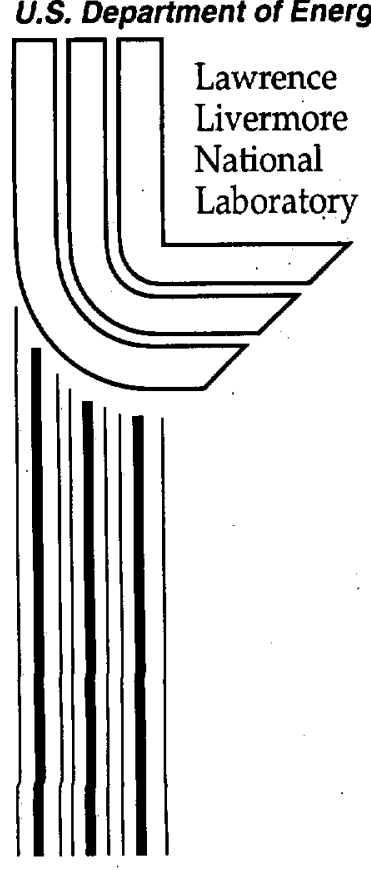




\section{DISCLAIMER}

This document was prepared as an account of work sponsored by an agency of the United States Government. Neither the United States Government nor the University of California nor any of their employees, makes any warranty, express or implied, or assumes any legal liability or responsibility for the accuracy, completeness, or usefulness of any information, apparatus, product, or process disclosed, or represents that its use would not infringe privately owned rights. Reference herein to any specific commercial product, process, or service by trade name, trademark, manufacturer, or otherwise, does not necessarily constitute or imply its endorsement, recommendation, or favoring by the United States Government or the University of California. The views and opinions of authors expressed herein do not necessarily state or reflect those of the United States Government or the University of California, and shall not be used for advertising or product endorsement purposes.

This is a preprint of a paper intended for publication in a journal or proceedings. Since changes may be made before publication, this preprint is made available with the understanding that it will not be cited or reproduced without the permission of the author.

This report has been reproduced directly from the best available copy.

Available electronically at http://www.doe.gov/bridge

Available for a processing fee to U.S. Department of Energy and its contractors in paper from

U.S. Department of Energy Office of Scientific and Technical Information

P.O. Box 62

Oak Ridge, TN 37831-0062

Telephone: (865) 576-8401

Facsimile: (865) 576-5728

E-mail: reports@adonis.osti.gov

Available for the sale to the public from

U.S. Department of Commerce

National Technical Information Service

5285 Port Royal Road

Springfield, VA 22161

Telephone: (800) 553-6847

Facsimile: (703) 605-6900

E-mail: orders@ntis.fedworld.gov

Online ordering: http://www.ntis.gov/ordering.htm

OR

Lawrence Livermore National Laboratory

Technical Information Department's Digital Library

http://www.llnl.gov/tid/Library.html 


\begin{tabular}{ccc}
\multicolumn{2}{c}{ Statistical Modeling of Large-Scale Simulation Data } \\
Tina Eliassi-Rad & Terence Critchlow & Ghaleb Abdulla \\
Center for Applied Scientific & Center for Applied Scientific & Center for Applied Scientific \\
Computing, Lawrence Livermore & Computing, Lawrence Livermore & Computing, Lawrence Livermore \\
National Laboratory & National Laboratory & National Laboratory \\
Livermore, CA 94551 & Livermore, CA 94551 & Livermore, CA 94551 \\
$+1(925) 422-1552$ & $+1(925) 423-5682$ & $+1(925) 423-5947$ \\
eliassi@llnl.gov & critchlow@\|nl.gov & abdulla1@\|lnl.gov
\end{tabular}

\begin{abstract}
With the advent of fast computer systems, scientists are now able to generate terabytes of simulation data. Unfortunately, the shear size of these data sets has made efficient exploration of them impossible. To aid scientists in gathering knowledge from their simulation data, we have developed an ad-hoc query infrastructure. Our system, called AQSim (short for Ad-hoc Queries for Simulation) reduces the data storage requirements and access times in two stages. First, it creates and stores mathematical and statistical models of the data. Second, it evaluates queries on the models of the data instead of on the entire data set. In this paper, we present two simple but highly effective statistical modeling techniques for simulation data. Our first modeling technique computes the true mean of systematic partitions of the data. It makes no assumptions about the distribution of the data and uses a variant of the root mean square error to evaluate a model. In our second statistical modeling technique, we use the Andersen-Darling goodness-of-fit method on systematic partitions of the data. This second method evaluates a model by how well it passes the normality test on the data. Both of our statistical models summarize the data so as to answer range queries in the most effective way. We calculate precision on an answer to a query by scaling the one-sided Chebyshev Inequalities with the original mesh's topology. Our experimental evaluations on two scientific simulation data sets illustrate the value of using these statistical modeling techniques on large simulation data sets.
\end{abstract}

\section{Categories and Subject Descriptors}

E.4 [Data]: Coding and Information Theory - data compaction and compression. G.3 [Mathematics of Computing]: Probability and Statistics - distribution functions, multivariate statistics. nonparametric statistics, statistical computing. H.2.4 [Database Management]: Systems - query processing. H.2.8 [Database Management]: Database Applications - data mining, scientific databases. H.3.1 [Information Storage and Retrieval]: Content Analysis and Indexing - indexing methods.

\section{General Terms}

Algorithms, Management, Measurement, Performance, Experimentation.

\section{Keywords}

statistical modeling, large-scale scientific data sets, approximate ad-hoc queries.

\section{INTRODUCTION}

Scientific experiments ran on the latest super computers are producing large-scale simulation data. The size of these data sets is typically on the order of terabytes, which makes even the best visualization tools inadequate. The need to efficiently explore these large simulation data sets has led to a surge of interest in scalable modeling and visualization tools [1][2][3][4][7][9].

In the DataFoundry Project, we have created a systen, called AQSim (short for Ad-hoc Queries for Simulation). Figure 1 illustrates AQSim's two processors. The first processor (a.k.a, model generator) builds statistical and mathematical models of the data. Subsequently, the second processor (a.k.a, query processor) executes user queries on the generated models to explore the data set.

Since most scientific simulation code generate mesh data, AQSim uses data in mesh format to build its models. A mesh data set consists of interconnected grids of small zones (see Figure 2). Data points are stored in the zones. Mesh data sets usually vary with time, contain multiple dimensions (i.e., variables), and have a huge number of irregular grids. Musick and Critchlow provide a nice introduction to scientific mesh data [3].

The main advantages of AQSim are two-folds. First, the model generator reduces the data storage requirements since models take less space than the original data set, which typically resides on tertiary storage. Second, the query processor decreases the access times since models of the data are queried.
Permission to make digital or hard copies of all or part of this work for personal or classroom use is granted without fee provided that copies are not made or distributed for profit or commercial advantage and that copies bear this notice and the full citation on the first page. To copy otherwise, or republish, to post on servers or to redistribute to lists, requires prior specific permission and/or a fee.

$K D D^{\prime}$ '02, July 23-26, 2002, Edmonton, Alberta, Canada.

Copyright 2002 ACM I-58113-000-0/00/0000 ..\$5.00. 


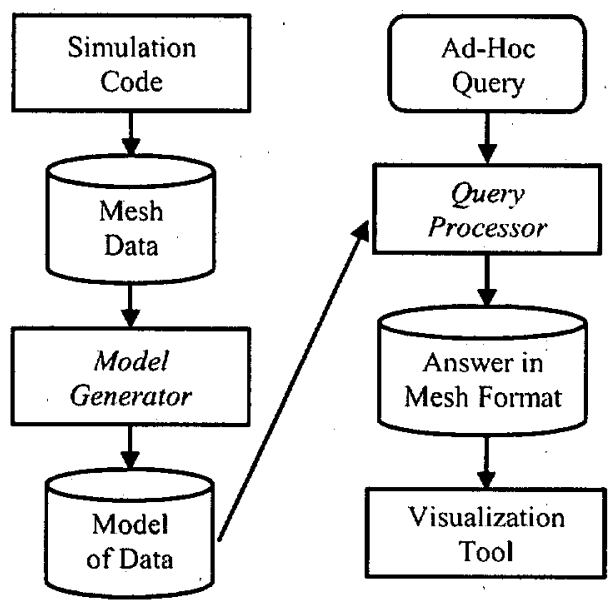

Figure 1. AQSIM Architecture

In this paper, we describe and evaluate two statistical modeling techniques for $\mathrm{AQSim}$. The first model captures the true mean of systematic partitions of the data. We call this model the mean modeler. The error on this model is a variant of the root mean square error (RMSE). The main advantages of mean modeler are as follows: (i) it makes no assumptions about the distribution of the data, and (ii) it calculated its model parameters through one sweep of the data. Our second model captures the normality of systentatic partitions of the data by utilizing the Anderson-Darling goodness-of-fit test [5]. This model is called the goodness-of-fit modeler. The error on this model is the Type I error associated with the goodness-of-fit test.

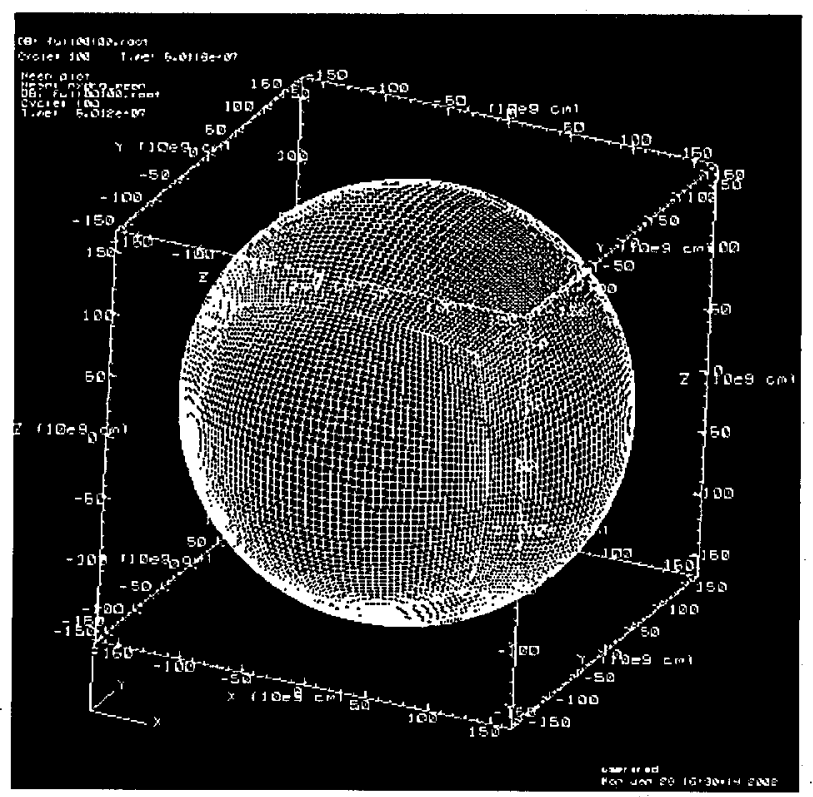

Figure 2. A Mesh Data Set Representing a Star

Despite their simplicity, these models have performed extremely well on our empirical studies of range queries. The answer to a query is judged by its precision to the original data. We calculate precision associated with a query's answer by scaling the onesided Chebyshev inequality with the original mesh topology.

In the next section, we will describe our modeling algorithms. Then, we will present two case-studies, which illustrate the value of our modeling techniques, in section 3 . We follow that with a discussion of some related, current, and future work. Finally, the paper concludes with some final remarks.

\section{AQSIM'S MODEL GENERATOR}

AQSim's model generator systematically partitions the data and builds models on each partition. This section describes two partitioning strategies and two statistical modeling techniques for AQSim.

\subsection{Partitioning Strategies}

AQSim's model generator builds models on partitions of the original data. Partitioning stops when models are accurate within a user-defined error threshold.

AQSim has two distinct partitioning strategies. The first is a topdown approach, where the data is divided in a four-way bisection on the spatial-temporal space (see Figure 3). The second approach is a bottom-up strategy, where the data points are conglomerated based on their zones in the mesh topology (see Figure 4).

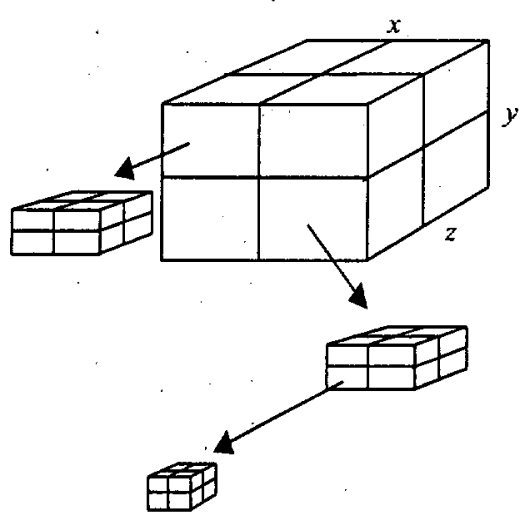

Figure 3. Top-Down Partitioning of the Data at a Particular Time Step

Table I summarizes the advantages of the two approaches. The bottom-up strategy is preferred over the top-down approach since it is cheaper computationally and it captures the mesh topology.

Table 1. Properties of AQSim's Partitioning Strategies

\begin{tabular}{|c|c|c|}
\hline Strategies & Bottom-Up & Top-Down \\
\hline $\begin{array}{c}\text { Mesh Topology } \\
\text { Captured }\end{array}$ & Yes & No \\
\hline $\begin{array}{c}\text { Computationally } \\
\text { Expensive }\end{array}$ & $\mathrm{O}\left(N_{\text {dasa }} \times \log \left(N_{\text {level }}\right)\right)$ & $\mathrm{O}\left(N_{\text {dara }} \times N_{\text {level }}\right)$ \\
\hline
\end{tabular}




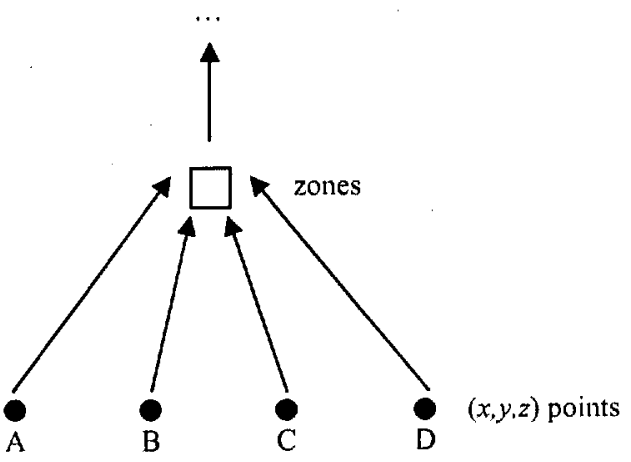

Figure 4. Bottom-Up Partitioning of the Data at a Particular Time Step

\subsection{Mean Modeler}

Each partition of the data has a set of variables associated with it. For each variable $\nu_{i}$, the mean modeler is $\mu_{i}$, where $\mu_{i}$ is the mean of the data points associated with $v_{i}$ in partition $p_{k}$.

For the mean modeler, partitioning of the data stops when either one of the following two conditions is true:

1. $\forall v \in$ NonPartitioningVariables in node $\eta, \sigma_{v}=0$.

2. $\forall v \in$ NonPartitioningVariables in node $\eta$, $\left(\mu_{v}-c . \sigma_{v} \leq \min _{v}\right) \&\left(\max _{v} \leq \mu_{v}+c . \sigma_{v}\right)$.

The first stopping criterion represents the simple case of partitions with either 1 data point or a set of data points with standard deviation of zero. In the second stopping criterion, the partition threshold, $c$, is a real number greater than or equal to zero. This user-defined threshold is a scaling factor for the standard deviation of variable $v$. For example, $c=1$ means that the minimum and maximum values for each non-partitioning variable must be within 1 standard deviation of the mean of the data points in the node. The advantage of the above stopping criteria is that it does not assume any distribution on the data points.

For the mean modeler, standard deviation is the same as RMSE (root mean square error) since the true mean, which is an unbiased estimator, is used as the model. The RMSE represents the error associated with the mean modeler.

\subsection{Goodness-of-Fit Modeler}

For each variable $v_{i}$ in partition $p_{k}$, the goodness-of-modeler is $\mathrm{N}\left(\mu_{i}, \sigma_{i}\right)$. That is, the model for $v_{i}$ is a normal distribution with mean, $\mu_{i}$, and standard deviation, $\sigma_{i}$

For the goodness-of-fit modeler, partitioning stops when the hypothesis test for normality is not rejected. We use the Anderson-Darling test for normality (which is considered to be the most powerful goodness-of-fit test for normality) for our goodness-of-fit test [5].

The Anderson-Darling test involves calculating the $A^{2}$ metric for variable $\nu_{i} \sim \mathrm{N}\left(\mu_{i}, \sigma_{i}\right)$, which is defined to be

$$
A^{2}=-\frac{1}{n}\left(\sum_{j=1}^{n}(2 j-1)\left(\ln \left(z_{j}\right)+\ln \left(1-z_{n+1-j}\right)\right)\right)-n
$$

where $n=$ number of data points for $v_{i}$ and $z_{j}=\Phi\left(\frac{x_{j}-\mu_{i}}{\sigma_{i}}\right)$. $\Phi(\cdot)$ is the standard nomal distribution function.

We reject $H_{0}$ if $A^{2}\left(1+\frac{0.75}{n}+\frac{2.25}{n^{2}}\right)$ exceeds the critical value associated with the user-specified error threshold. Otherwise, we accept $H_{0}$.

For each variable $v_{i}$, the error on this model is defined to be $\operatorname{Pr}$ (reject $H_{0} / H_{0}$ is true), where $H_{0}$ is the null hypothesis and states that the distribution of a variable $V_{1}$ is normal. In other words, the model error is equal to the Type I error.

\section{AQSIM's QUERY PROCESSOR}

AQSim's query processor takes a user's query and a value for the amount of time that the user is willing to wait for an answer. Then, while its running time is less than the user's constraint, the query processor searches the hierarchical partitions (which were made by the model generator) for the partitions that answer the user's query with the highest precision.

Precision $\left(Q_{u s e}\right.$, model $_{j}$, partition $\left._{i}\right)$ is defined to be the precision of

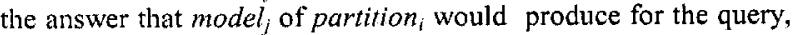
$Q_{u s e r}$, as a percentage of partition ${ }_{i}$ 's mesh topologv. Specifically, Precision $\left(Q_{u s e r}\right.$, model $_{j}$, partition $\left._{i}\right)=\left(\right.$ partition $_{i} \rightarrow$ filled_volume $) \times$ $P\left(Q_{u s e r}\right.$, model $_{i}$, parition $\left._{i}\right)$, where filled_volume corresponds to the percentage of non-empty space in the partition's spatial bounding $b o x$ and is defined to be

$$
\begin{aligned}
& \text { \# of children } \\
& \sum\left(\text { filled_volume } e_{\text {child }} \times \text { volume }_{\text {child }}\right) \\
& \text { filled_volume }_{\text {parent }}=\frac{\text { child }=1}{\text { volume }_{\text {parent }}}
\end{aligned}
$$

$P\left(Q_{u s e r}\right.$, model $_{j}$, node $\left.i\right)$ is calculated by using the one-sided Chebyshev inequalities [6], which are defined to be

$$
\begin{aligned}
& P(X \leq \mu-\alpha) \leq \frac{\sigma^{2}}{\sigma^{2}+\alpha^{2}} \\
& \text { - } P(X \geq \mu+\alpha) \leq \frac{\sigma^{2}}{\sigma^{2}+\alpha^{2}}
\end{aligned}
$$

Here is a simple example of how precision is calculated. Suppose we are given the following query, pressure $\leq 0.5$. Then, for a partition, say $p$, the precision is equal to

Precision(pressure $\leq 0.5$, mean modeler, $p)=(p \rightarrow$ filled_volume $)$

$\times P($ pressure $\leq 0.5)=(p \rightarrow$ filled_volume $) \times P\left(\right.$ pressure $\leq \mu_{\text {pressure }}-$

$\alpha) \leq(p \rightarrow$ filled_volume $) \times \frac{\sigma_{\text {pressure }}^{2}}{\sigma_{\text {pressure }}^{2}+\left(\mu_{\text {pressure }}-0.5\right)^{2}}$, where $\alpha$

$=\mu_{E Q P S}-0.5$.

For more complicated queries, we make new random variables and calculate mean and standard deviations for them based on the original mean and standard deviations. The advantage of using the Chebyshev inequalities is that no assumption is made on the distribution of the data in a node. 


\section{EXPERIMENTAL EVALUATION 4.1 The Can Data Set}

Our first data set represents a wall crushing a can. It has 14 variables, ${ }^{1} 44$ time steps, and 443,872 data points. Figure 5 depicts this data set in its first time step when all the points $440 \mathrm{~K}$ points are plotted.

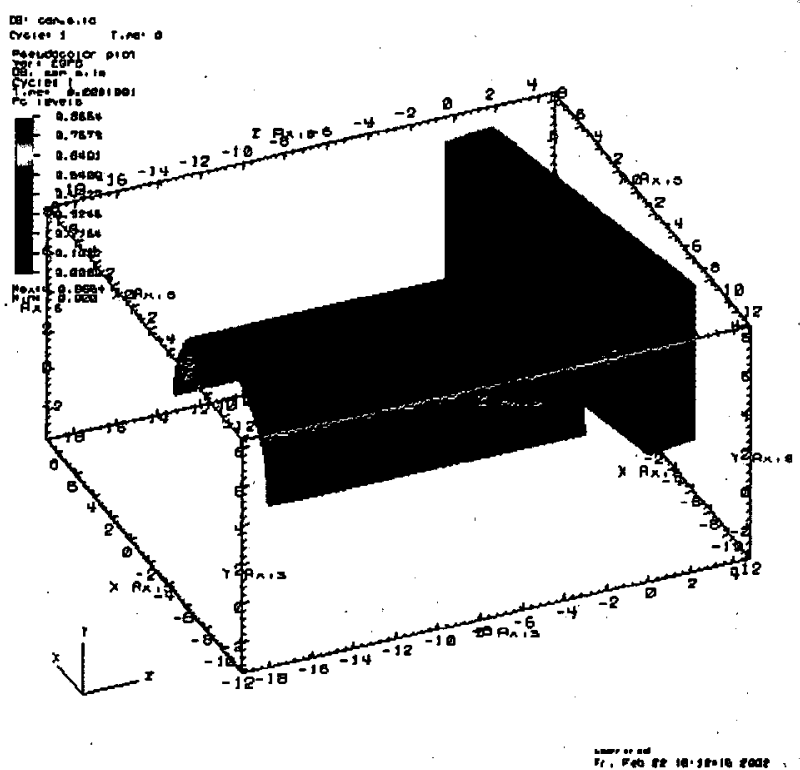

Figure 5. Can Data Set at its First Time Step

Table 2 lists the compression results on the can data for the mean modeler. Recall that for the partition threshold for this modeler restricts the distance between minimum and maximum values of a variable and its mean value with respect to RMSE.

Table 2. Mean Modeler's Compression Results on the Can Data

\begin{tabular}{|c|c|c|c|c|c|}
\hline $\begin{array}{c}\text { Partition } \\
\text { Threshold }\end{array}$ & $\begin{array}{c}\text { \% of } \\
\text { Compression }\end{array}$ & Total \# of & $\begin{array}{c}\% \text { of non- } \\
\text { leaf } \\
\text { partitions }\end{array}$ & $\begin{array}{c}\text { Avg. \# of leaf } \\
\text { partitions }\end{array}$ & $\begin{array}{c}\text { data point } \\
\text { in a } \\
\text { partition }\end{array}$ \\
\hline 1.00 & 4.2 & 425,075 & 19.4 & 80.6 & 1.3 \\
\hline 1.50 & 33.0 & 297,566 & 13.4 & 86.6 & 1.7 \\
\hline 1.75 & 40.1 & 265,939 & 12.5 & 87.5 & 1.9 \\
\hline 2.00 & 51.5 & 215,255 & 11.1 & 88.9 & 2.3 \\
\hline 2.25 & 62.4 & 166,986 & 10.3 & 89.7 & 3.0 \\
\hline 2.50 & 71.6 & 125,912 & 9.6 & 90.4 & 3.9 \\
\hline 2.75 & 78.1 & 97,410 & 9.1 & 90.9 & 5.0 \\
\hline 3.00 & 82.6 & 77,277 & 8.6 & 91.4 & 6.3 \\
\hline
\end{tabular}

I The can data set's variables are as follows: time, $x$ axis, $y$ axis, $z$. axis, pressure, acceleration in $\mathrm{x}$ axis, acceleration in $\mathrm{y}$ axis, acceleration in $z$ axis, velocity in $x$ axis, velocity in $y$ axis, velocity in $z$, displacement in $x$ axis, displacement in $y$ axis, displacement in $\mathbf{z}$ axis
For our mean modeler experiments, Figures 6 through 8 show the can data set at its first time step when the query time $>0$ is posed with no constraint on execution time (that is precision equals $100 \%$ ) and with partition thresholds of $1.00,2.00$, and 3.00 , respectively. As expected, we get better compression as the partition threshold for the mean modeler gets larger (since we are allowing the range of values for a variable to me larger). However, as you see in Figure 8 even with $82.6 \%$ compression, we are able to return an answer with $100 \%$ precision.

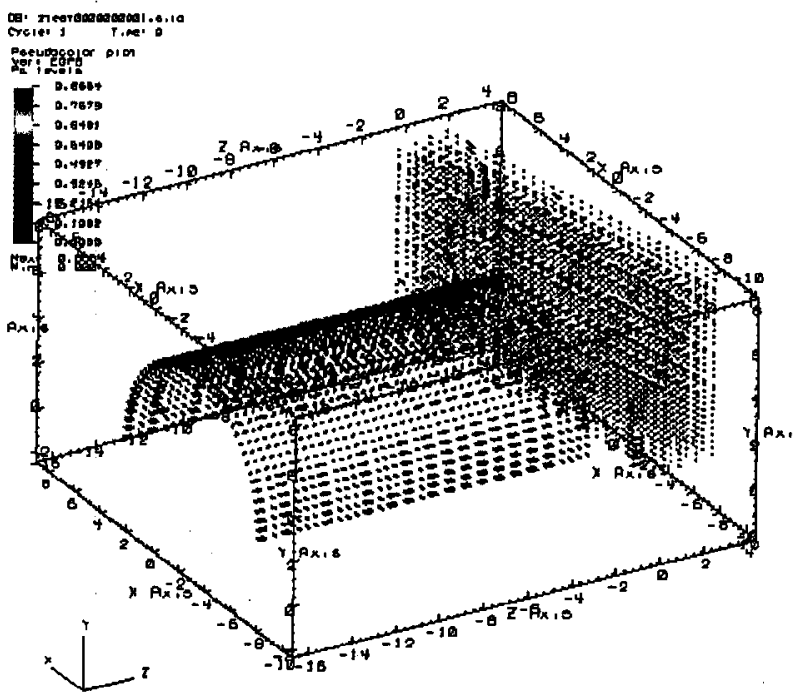

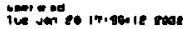

Figure 6. Can Data Set at its First Time Step with Partition Threshold of 1.00, Query Time $>0$, and Precision $=100 \%$

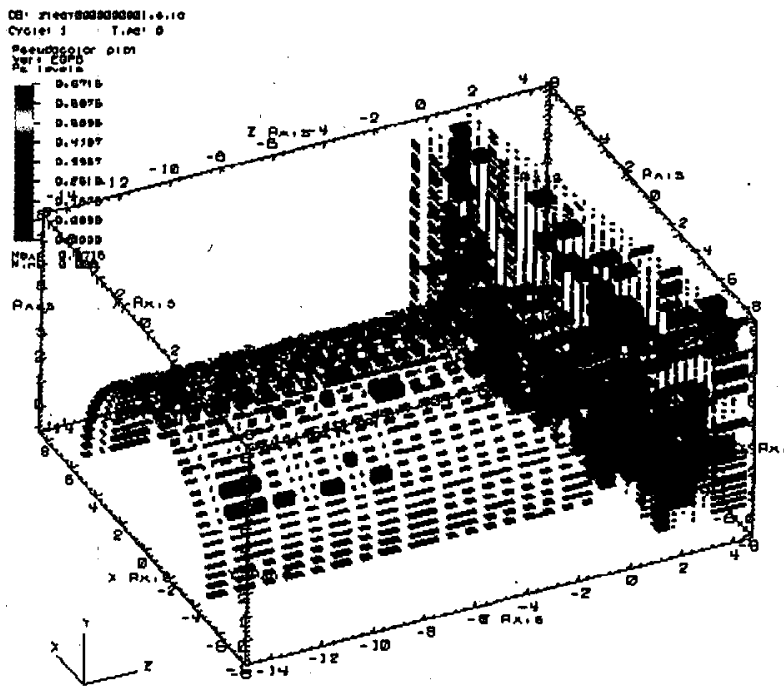

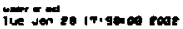

Figure 7. Can Data Set at its First Time Step with Partition Threshold of 2.00, Query Time $>0$, and Precision $=100 \%$ 


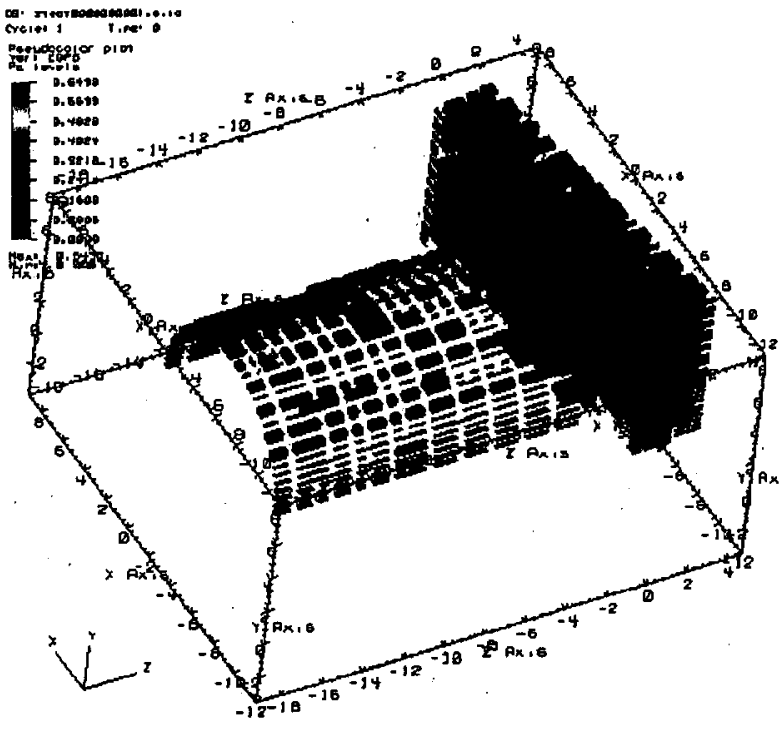

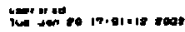

Figure 8. Can Data Set at its First Time Step with Partition Threshold of 3.00, Query Time $>0$, and Precision $=100 \%$

Table 3 lists the compression results on the can data for the goodness-of-fit modeler. The partition threshold in this table represents the confidence region of our normality test, which is equal to $100 \times(1-$ Type I error $)$.

Table 3. Goodness-of-Fit Modeler's Compression Results on the Can Data

\begin{tabular}{|c|c|c|c|c|c|}
\hline $\begin{array}{c}\% \\
\text { Partition } \\
\text { Threshold }\end{array}$ & $\begin{array}{c}\text { \% of } \\
\text { Compression }\end{array}$ & $\begin{array}{c}\text { Total \# of } \\
\text { partitions }\end{array}$ & $\begin{array}{c}\% \text { of non- } \\
\text { leaf } \\
\text { partitions }\end{array}$ & $\begin{array}{c}\text { \% of leaf } \\
\text { partitions }\end{array}$ & $\begin{array}{c}\text { Avg. \# of } \\
\text { data point } \\
\text { in a } \\
\text { partition }\end{array}$ \\
\hline 50.0 & 39.6 & 272,583 & 12.6 & 87.4 & 1.9 \\
\hline 80.0 & 57.3 & 189,533 & 10.1 & 89.9 & 2.6 \\
\hline 85 & 60.9 & 173,766 & 9.7 & 90.3 & 2.8 \\
\hline 90.0 & 65.8 & 151,818 & 9.3 & 90.7 & 3.2 \\
\hline 95.0 & 73.7 & 116,948 & 8.8 & 91.2 & 4.2 \\
\hline 99.99 & 91.4 & 38,344 & 7.3 & 92.7 & 12.5 \\
\hline
\end{tabular}

For our goodness-of-fit modeler experiments, Figures 9 through 11 show the can data set at its first time step when the query time $>0$ is posed with no constraint on execution time (that is precision equals $100 \%$ ) and with partition thresholds of $99.99 \%$, $95 \%$, and $50 \%$ respectively. Again not surprisingly, we get better compression as the partition threshold for the goodness-of-fit modeler gets larger (since the confidence region shrinks). However, as you see in Figure 11 even with $91.4 \%$ compression, we are able to return an answer with $100 \%$ precision.

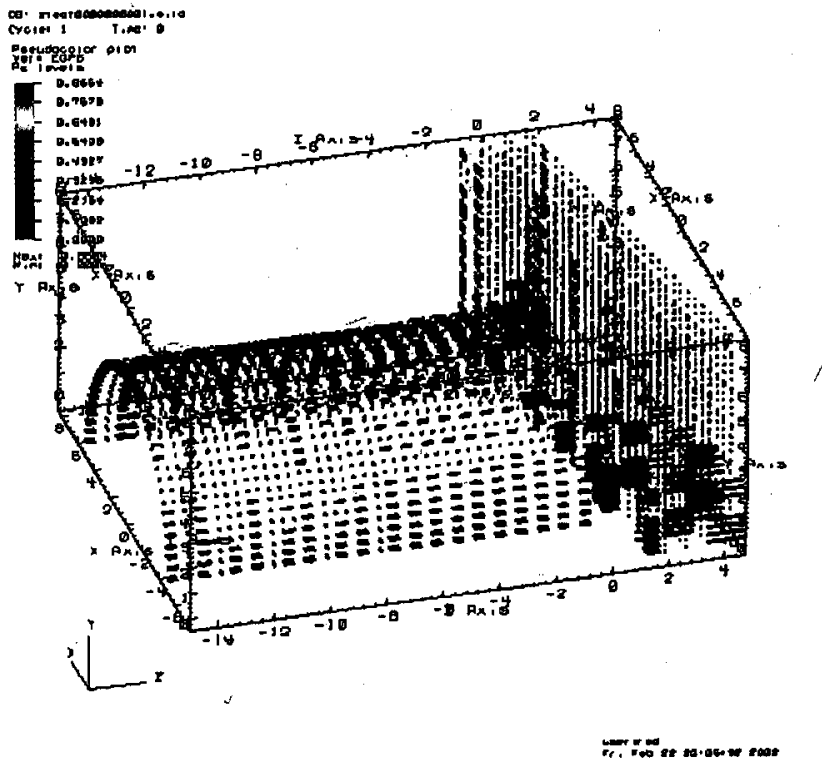

Figure 9. Can Data Set at its First Time Step with Partition Threshold of 50\%, Query Time $>0$, and Precision $=100 \%$

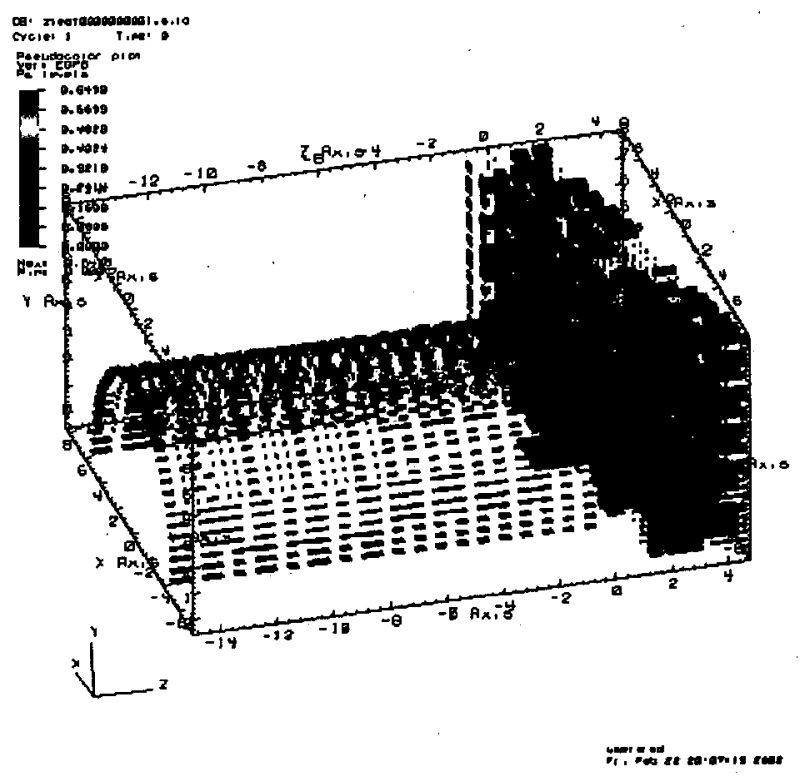

Figure 10. Can Data Set at its First Time Step with Partition Threshold of $95 \%$, Query Time $>0$, and Precision $=100 \%$ 


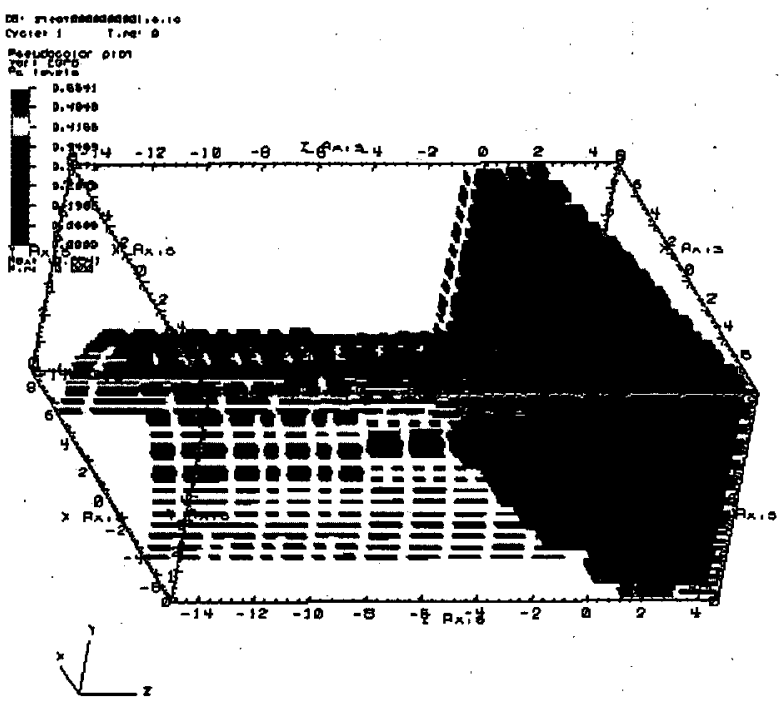

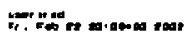

Figure 11. Can Data Set at its First Time Step with Partition Threshold of $99.99 \%$, Query Time $>0$, and Precision $=100 \%$

\subsection{The Astrophysics Data Set}

Our second data set represents a star in its mid-life. It has 18 variables, ${ }^{2} 16$ time steps, and 1,708,852 zones. Figure 12 depicts this data set in its first time step when all the points 1.7 million points are plotted.

Table 4 lists the compression results on the astrophysics data for the mean modeler. Again, recall that the partition threshold for this modeler restricts the distance between minimum and maxinum of a variable and its mean value with respect to RMSE.

For our mean modeler experiments, Figure 13 shows the can data set at its first time step when the query time $>0$ is posed with no constraint on execution time (that is precision equals $100 \%$ ) and with partition thresholds of 3.00. Similar to our experiments on the can data set, we get better compression as the partition threshold for the mean modeler gets larger (since we are allowing the range of values for a variable to me larger). However, as you see even with $92.1 \%$ compression, we are able to return an answer with $100 \%$ precision.

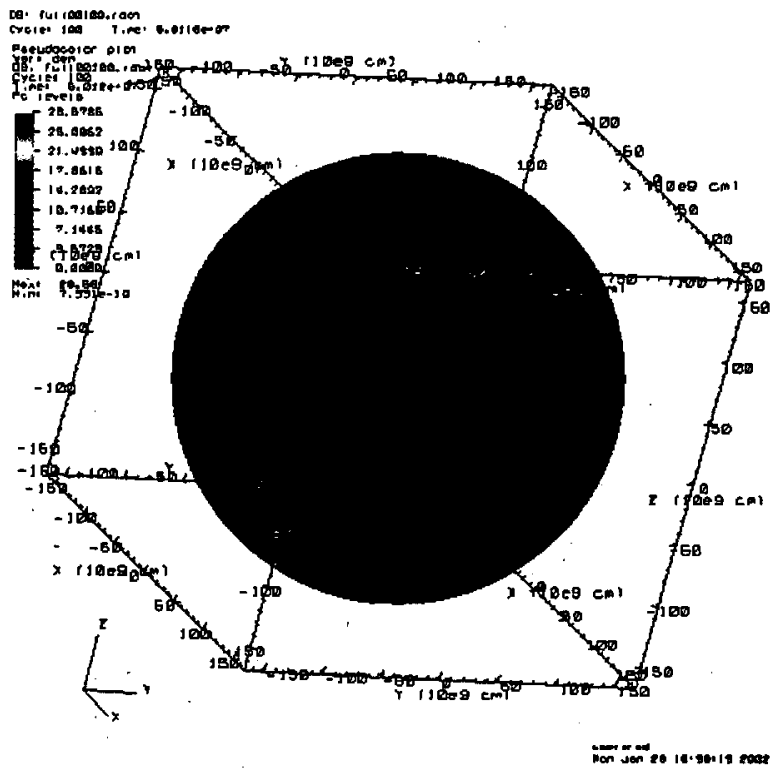

Figure 12. Astrophysics Data Set at its First Time Step

Table 4. Mean Modelers' Compression Results on the Astrophysics Data

\begin{tabular}{|c|c|c|c|c|c|}
\hline $\begin{array}{c}\text { Partition } \\
\text { Threshold }\end{array}$ & $\begin{array}{c}\text { \% of } \\
\text { Compression }\end{array}$ & $\begin{array}{c}\text { Total \# of } \\
\text { partitions }\end{array}$ & $\begin{array}{c}\text { \% of non- } \\
\text { leaf } \\
\text { partitions }\end{array}$ & $\begin{array}{c}\text { \% of leaf } \\
\text { partitions }\end{array}$ & $\begin{array}{c}\text { Avg. \# of } \\
\text { data point } \\
\text { in a } \\
\text { partition }\end{array}$ \\
\hline 1.75 & 67.4 & 728,081 & 17.9 & 82.1 & 2.9 \\
\hline 2.00 & 70.1 & 511,395 & 17.8 & 82.2 & 4.1 \\
\hline 2.25 & 79.7 & 347,471 & 17.7 & 82.3 & 6.0 \\
\hline 2.50 & 85.8 & 242,840 & 18.7 & 81.3 & 8.7 \\
\hline 2.75 & 89.6 & 177,448 & 19.0 & 81.0 & 11.9 \\
\hline 3.00 & 92.1 & 135,548 & 17.8 & 82.2 & 15.3 \\
\hline
\end{tabular}

\footnotetext{
${ }^{2}$ The astrophysics data set's variables are as follows: time, $x$ axis, $\mathrm{y}$ axis, $\mathrm{z}$ axis, distance, grid vertex values, grid movement in $\mathrm{x}$ axis, grid novenent in y axis, $d$ (energy)/d(temperature), density, electron temperature, temperature due to radiation, pressure, artificial viscosity, material temperature, material velocity in $x$ axis, material velocity in y axis, material velocity in $\mathrm{z}$ axis.
} 


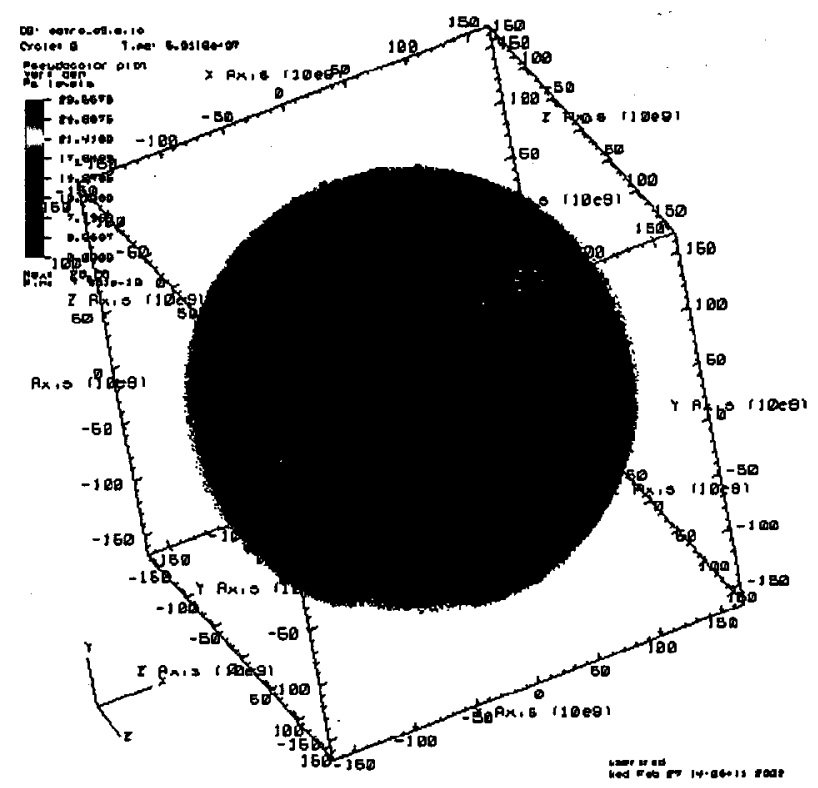

Figure 13. Astrophysics Data Set at its First Time Step with Partition Threshold of 3;00, Query Time $>0$, and Precision $=100 \%$

Table 5 lists the compression results on the can data for the goodness-of-fit modeler. Recall that the partition threshold in this table represents the confidence region of our normality test, which is equal to $100 \times(1-$ Type I error $)$.

Table 5. Goodness-of-Fit Modeler's Compression Results on the Astrophysics Data

\begin{tabular}{|c|c|c|c|c|c|}
\hline $\begin{array}{c}\% \\
\text { Partition } \\
\text { Threshold }\end{array}$ & $\begin{array}{c}\% \text { of } \\
\text { Compression }\end{array}$ & Total \# of & $\begin{array}{c}\text { \% of non- } \\
\text { leaf } \\
\text { partitions }\end{array}$ & $\begin{array}{c}\text { \%vo of leaf } \\
\text { partitions }\end{array}$ & $\begin{array}{c}\text { data point } \\
\text { in a } \\
\text { partition }\end{array}$ \\
\hline 80.0 & 66.7 & 564,718 & 16.8 & 83.2 & 3.6 \\
\hline 85 & 71.2 & 492,029 & 16.7 & 83.3 & 4.2 \\
\hline 90.0 & 76.4 & 404,136 & 16.9 & 83.1 & 5.1 \\
\hline 95.0 & 82.8 & 293,585 & 16.8 & 83.2 & 7.0 \\
\hline 99.99 & 94.3 & 97,819 & 13.3 & 86.7 & 20.2 \\
\hline
\end{tabular}

For our goodness-of-fit modeler experiments, Figures 14 shows the astrophysics data set at its first time step when the query time $>0$ is posed with no constraint on execution time (that is precision equals $100 \%$ ) and with partition thresholds of $99.99 \%$. Again not surprisingly, we get better compression as the partition threshold for the goodness-of-fit modeler gets larger (since the confidence region shrinks). However, as you see in Figure 14 even with $94.3 \%$ compression, we are able to return an answer with $100 \%$ precision.

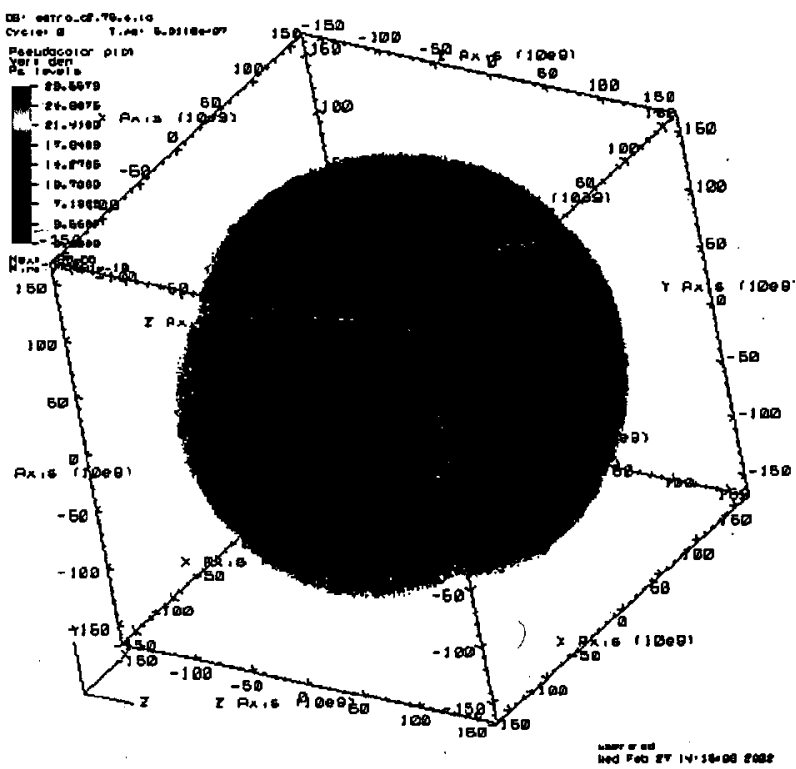

Figure 14. Astrophysics Data Set at its First Time Step with Partition Threshold of $99.99 \%$, Query Time $>0$, and Precision $=100 \%$

\subsection{Discussion}

Our experimental results illustrate the value of using simple statistical modeling techniques on scientific simulation data sets. Both of our approaches require only one sweep of the data and generate models that compress the data up to $94 \%$.

The goodness-of-fit modeler performed better than the mean modeler on the two data sets presented in this paper. This is not surprising to us since our two data sets describe physical phenomena and the goodness-of-fit modeler is biased towards such normally distributed data sets. In general, we prefer the mean modeler since it makes no assumption on the data.

\section{RELATED WORK}

Our work is similar to Freitag and Loy's work at Argonne National Laboratory [7]. Their system builds distributed octrees from large scientific data sets. They, however, reduce their data by constraining the points to their spatial locations. They also don't allow the user to query the octree. Instead, the user can view the tree at different resolutions.

STING [9] is also similar to AQSim except that it assumes that the distribution of the data is known. It has also been tested only on small data sets containing only tens of thousands of data points.

AQUA [2] uses cached summary data in an OLAP domain. Unfortunately, they using sampling and histogram techniques, which are not good for scientific data sets because by sampling you night miss outliers (which are important in scientific data sets) and histograms are computationally expensive when you have high dimensional data. 


\section{CURRENT AND FUTURE WORK}

We are investigating other modeling techniques for AQSim's model generator. Specifically, we are constrained to models that (i) require only one sweep of data, (ii) are good at finding outliers, (iii) can be easily parallelized, and (iv) can efficiently answer nonrange queries (see [3])

We are also interested in optimal disk layout of the index tree. In particular, we are investigating techniques which will minimize seek time. Moreover, parallelizing AQSim's query processor is also part of our future work. Finally, we are conducting experiments on other larger data sets.

\section{CONCULSION}

To help scientists in gathering knowledge from their large-scale simulation data, we have developed an ad-hoc query infrastructure, called AQSim. Our system reduces the data storage requirements and access times in two stages. First, it creates and stores mathematical and statistical models of the data. Second, it evaluates queries on the models of the data instead of on the entire data set. In this paper, we present two simple but highly effective statistical modeling techniques for simulation data. Our first modeling technique computes the true mean of systematic partitions of the data. It makes no assumptions about the distribution of the data and uses a variant of the root mean square error to evaluate a model. In our second statistical modeling technique, we use the Andersen-Darling goodness-of-fit method on systematic partitions of the data. This second method evaluates a model by how well it passes the normality test on the data: Both of our statistical models summarize the data so as to answer range queries in the most effective way. We calculate precision on an answer to a query by scaling the one-sided Chebyshev Inequalities with the original mesh's topology. Our experimental evaluations on two scientific simulation data sets illustrate the value of using these statistical modeling techniques on large simulation data sets.

\section{ACKNOWLEDGMENTS}

Our thanks to Chuck Baldwin, Kevin Durrenberger, Roy. Kamimura, Ed Smith, and Nu Ai Tang for their useful comments and assistance.

\section{REFERENCES}

[1] Abdulla, G., Baldwin, C., Critchlow, T., Kamimura, R., Lozares, l., Musick, R., Tang, N.A., Lee, B., and Snapp, R. Approximate ad-hoc query engine for simulation data. In Proceedings of JCDL 2001 (Roanoke VA, June 2001), ACM Press, 255-256.

[2] Acharya, S., Gibbsons, P.B., Poosala, V., and Ramaswamy, $\mathrm{S}$. The Aqua approximate query answering system. In Proceedings of the 1999 ACM SIGMOD, ACM Press, 574 576.

[3] Baldwin, C., Abdulla, G., and Critchlow, T. MultiResolution Modeling of Large Scale Scientific Sinulation Data. LLNL Technical Report, 2002.

[4] Chakrabarti, K., Garofalakis, M., Rastogi, R., and Shim, K Approximate query processing using wavelets, In Proceedings of VLDB 2000 (Cairo Egypt, September 2000), ACM Press, 111-122.

[5] D'Agostino, R.B., and Stephens, M.A. Goodness-of-fit Techniques, Marcel Dekker, Inc., 1986.

[6] Devore, J.L. Probability and Statistics for Engineering and the Sciencfes, 3rd edition. Brooks/Cole Publishing Company, Pacific Grove, CA, 1991.

[7] Freitag, L.A., and Loy, R.M. Adaptive, multiresolution visualization of large data sets using a distributed memory octree. In Proceedings of SC 1999 (Portland OR, November 1999), ACM Press, Article 60.

[8] Musick, R., and Critchlow, T. Practical lessons in supporting large-scale computational science, In Proceedings of SIGMOD Record 1999, ACM Press, 28(4):49-57.

[9] Wang, W., Yang, J., and Muntz, R. STING: A statistical information grid approach to spatial data mining. In Proceedings of the VLDB (Athens Greece, August 1997), Morgan Kaufmann Publishers, 186-195. 